\title{
Naissance des relations entre justice et psychiatrie à Genève
}

\author{
Par Cléopâtre Montandon
}

On ne peut comprendre l'apparition de l'examen psychiatrique en matière pénale si on ne fait pas intervenir l'évolution du concept de responsabilité ${ }^{1}$. Dans les sociétés archaïques où l'on ne faisait pas de différence nette entre crime et trouble social, la procédure juridique n'avait pas comme objectif la punition des transgresseurs de normes mais le rétablissement de l'harmonie sociale, la réconciliation des parties adverses. Dans les groupes sociaux où chaque individu dépendait pour sa survie quotidienne de l'aide de ses compagnons, où les relations étaient celles d'une interdépendance mutuelle, la responsabilité individuelle n'avait pas de sens. Les individus qui transgressaient les lois ne devaient pas répondre pour leurs fautes, leurs péchés, leurs crimes en soi, mais devaient répondre de leurs actes envers la victime, le clan, le groupe social lésé. La responsabilité n'était pas subjective mais objective. On ne jugeait pas en tenant compte de la faute subjectivement perçue mais de la faute dans ses résultats objectifs. Cela donnait parfois lieu à certaines sanctions envers des choses, des animaux, des petits enfants ou des fous qui peuvent nous apparaître aberrantes aujourd'hui mais qui rendaient possibles des actes de réparation et de restitution envers la victime. On a trop souvent critiqué, se basant sur des applications simplistes, le principe de cette responsabilité objective qui a toutefois le mérite de permettre la considération d'une responsabilité collective.

Les sociétés occidentales ont été influencées par la notion de la faute morale du droit romano-chrétien, elles ont individualisé, subjectivisé la responsabilité, mais cela aboutit parfois à des répressions tout aussi odieuses que celles basées sur la responsabilité objective des sociétés primitives.

Les théories criminologiques du $\mathrm{XVIII}^{\mathrm{e}}$ siècle, imbues de principes humanitaires, ont réussi à adoucir le droit pénal, sans pour autant modifier le principe de la responsabilité individuelle. Ces théories préconisaient que les peines administrées soient mesurées et proportionnelles au crime. Elles recommandaient l'examen objectif de l'acte criminel, partant du principe qu'il existe chez tout criminel un raisonnement rationnel, ses actes étant libres et volontaires.

De ce fait, les théories appartenant à l'école criminologique classique à laquelle le Milanais Beccaria est rattaché (Des délits et des peines, 1764) évitaient. la discussion de la motivation de l'acte criminel.

Cependant, en pratique, les variations psychologiques et sociales entre les différents acteurs commençaient à préoccuper les juges. L’école criminologique néo- 
classique du $\mathrm{XIX}^{\mathrm{e}}$ siècle développa la notion des circonstances atténuantes. Les défenseurs de ce point de vue désiraient qu'on tienne compte des motivations des délinquants tout comme il était admis que certains individus, les enfants, les vieillards, les fous, les débiles, ne sont pas libres de leurs actes. Par ailleurs, les pénologues de cette école influencés par l'utilitarisme de Bentham ont également commencé à étudier les effets sur les criminels de cette forme de punition qui se généralisait, l'incarcération. Certains ont contribué à créer l'illusion qu'il suffisait de réformer les prisons pour réhabiliter les criminels. Ces nouvelles conceptions de l'école néo-classique ont ouvert la porte aux experts, notamment aux psychiatres.

Ce fut principalement l'école positiviste, dont l'un des objectifs principaux était l'explication scientifique de la criminalité, qui depuis la fin du $\mathrm{XIX}^{\mathrm{e}}$ siècle, attribua un rôle très important aux experts. Les positivistes, tel Lombroso (L'homme criminel, 1876), Ferri (La sociologie criminelle, 1881) et Garofallo (La criminologie, 1885), pensaient que le criminel se révèle par ses actes, qu'il subit des déterminismes biologiques, psychologiques et sociaux dont il n'est pas conscient, que le libre arbitre n'existe pas et que les criminels ne devraient pas être punis mais examinés et traités par des experts, entre autres des psychiatres. Ces derniers ont été vite séduits par l'idée qu'ils pourraient d'une part mieux faire comprendre l'acte criminel, et d'autre part contribuer à mieux traiter, voire « guérir » les criminels.

Ainsi, les médecins psychiatres ont participé à la médicalisation de la criminalité. Si certains de leurs précurseurs se sont efforcés de combattre l'idée que les fous étaient criminels, déjà à l'époque des procès de sorcellerie, d'autres tout au contraire ont contribué par la suite et notamment à la suite de Lombroso à faire croire que les criminels étaient fous.

On ne peut que se demander si l'interpénétration de la psychiatrie et de la justice est un effet de conditions historiques accidentelles et inhérentes à l'évolution de ces deux disciplines, ou s'il existe un rapport plus profond entre justice et psychiatrie qui est inscrit dans l'existence de jugements de valeur analogues sur la normalité.

Après ces considérations historiques générales, nous nous attacherons à décrire plus particulièrement la situation à Genève concernant les rapports entre psychiatrie et droit ainsi que le statut de criminel aliéné. Au XVIII ${ }^{\mathrm{e}}$ siècle, les aliénés étaient enfermés souvent enchaînés dans des maisons de discipline. A Genève, la «Discipline» était le lieu où l'on enfermait les pauvres, mendiants, vagabonds, délinquants et autres personnes en rupture avec le système social. Il en était de même pour les aliénés et à plus forte raison pour les délinquants aliénés. La 
«Discipline» (l'actuel bâtiment de Saint-Antoine) était une annexe de l'Hôpital général (l'actuel Palais de Justice), les deux institutions étant gérées par la même direction.

La décision qu'un délinquant arrêté était fou et ne devait pas être puni mais plutôt interné (quoique cet internement ne se distinguait guère de la punition), ne dépendait pas nécessairement de l'avis d'un médecin. Ainsi, en 1764, par exemple la décision qu'un détenu à la prison (probablement à l'Evêché) soit transféré à l'«hôpital des fous» (probablement la Discipline) est prise par le Procureur à la suite des plaintes d'un geôlier qui déclarait que ce détenu cassait et détruisait tout. Le Procureur, sans préavis médical, ordonna que Sieur S. soit «interdit et séquestré jusqu'à ce que les remèdes qu'on lui administre lui aient rendu l'usage de sa Raison», car dans le cas de ce détenu, il paraissait "presque impossible de concilier l'intérêt de la sûreté publique avec les égards qu'inspire l'humanité pour son état malheureux ${ }^{2}$. On trouve ici la préoccupation d'une virtualité dangereuse que les psychiatres vont reprendre plus tard.

Vers la fin du XVIII ${ }^{\mathrm{e}}$, on voit apparaître ce que l'on pourrait qualifier de premières ébauches d'expertises psychiatriques. Ainsi, en 1798, pour le cas d'une personne arrêtée parce qu'elle jetait ses meubles par la fenêtre, nous avons retrouvé le rapport d'un médecin. Ce dernier écrit:

«Je soussigné, Docteur en Médecine, déclare et atteste qu'ayant visité plusieurs fois le nommé Henry W., détenu actuellement à la maison de force, je l'ai trouvé dans un état d'aliénation d'esprit avec agitation, qui porte le caractère d'une imbecillité déjà invétérée et dont on ne peut espérer une amélioration, que du temps, d'une meilleure saison, et des remèdes qu'elle fournit. Les moyens répétés qu'on a déjà opposés à sa maladie sans succès, laissent toutefois à redouter qu'elle ne soit insurmontable. ${ }^{3}$

Si un détenu était reconnu aliéné, il était déclaré irresponsable et interné pour une période jugée utile par les médecins. Henry W. qui fut transféré à la Discipline a dû profiter des réformes d'Abraham Joly, médecin à l'hôpital et à la Discipline de Genève qui, avant Pinel, entre 1787 et 1792, a aboli les chaînes et les colliers de fer dont on se servait pour contenir les fous, les substituant par le corset de force en toîle ${ }^{4}$.

Il faut peut-être rappeler qu'à cette époque, la compétence d'interner les aliénés n'était pas nécessairement du ressort des médecins et ne le fut pas pendant très longtemps. Au XVIII ${ }^{\mathrm{e}}$ siècle, à la demande des familles, le Premier Syndic fournissait un billet ordonnant l'enfermement des individus récalcitrants ${ }^{5}$.

A partir du moment où Genève devint en 1798 chef-lieu du département du Léman, elle fut soumise aux lois françaises. Ainsi, en 1810, elle adopta l'article 64 du nouveau Code Pénal Français qui déclare qu'il n'y a «ni crime, ni délit lorsque 
le prévenu était en état de démence au temps de l'action ou lorsqu'il a été contraint par une force à laquelle il n'a pu résister».

Concernant les aliénés en général, en 1829, le Conseil d'Etat décréta que l'admission d'un aliéné dans un asile pouvait avoir lieu sous simple réquisition du Lieutenant de police. Le Procureur Général devait en prendre connaissance dans les vingt-quatre heures. Cela fut ratifié par la loi de 1838 sur le placement et la surveillance des aliénés qui donna la compétence d'interner au Département de justice et police. Ce n'est qu'à partir de 1936 que l'autorisation d'un internement est délivré sur vue d'un certificat médical par le Département de prévoyance sociale et de la santé publique.

Pour revenir à l'examen de l'état mental des inculpés, l'article 64 du code pénal français sous-entendait qu'il y avait démence lorsqu'il y avait délire, hallucinations, lorsque l'on décelait des troubles de l'entendement. Après la Restauration, le projet de Code Pénal pour la République et Canton de Genève de 1817 retient cette idée. La démence selon l'article 14 est un des cas où le fait en contravention à la loi n'est pas imputable à son auteur. L'article 16 spécifie: «La non-imputabilité d'un fait pour cause de démence s'applique à tous les cas où, à l'époque de l'acte, son auteur était par l'effet d'une maladie mentale, entièrement privé de l'usage de sa raison». Dans ce contexte, la tâche du psychiatre était assez simple. Ainsi, au sujet d'un homme de 33 ans qui avait tué sa femme, le jury genevois déclara qu'il était non-coupable et prononça en 1851 l'acquittement se basant sur le rapport du médecin. Le rapport des Drs d'Espine et Coindet disait à propos de cet homme au regard inquiet, mobile et vague :

«Nous ne mettons nullement en doute qu'il en soit atteint d'hallucinations du sens, du goût et de l'odorat qui lui ont fait croire que sa femme mêlait à des aliments des substances dégoûtantes ou délétères. De plus, il est sujet aux hallucinations du sens de l'ouïe. Il se plaint de railleries, d'insultes que lui adressaient au travers du mur de son appartement les personnes du voisinage, et même les enfants, des inconnus passant dans la rue. $\nu^{6}$

Cet homme avait déjà fait un séjour à la maison d'aliénés. C'est là qu'il fut probablement conduit après son acquittement. Il faut préciser que depuis 1832, les aliénés avaient été transférés de la Discipline à une maison située à Corsier sous la pression d'une épidémie de choléra qui encombra la Discipline. Gaspard de la Rive, grand défenseur des aliénés, obtint que ces derniers ne soient plus réintégrés à la Discipline ${ }^{7}$. Il écrivait que cette mesure serait odieuse pour le public:

«Rappelons le spectacle déchirant de ces femmes, de ces hommes, demi-nus, s'attachant aux barreaux de leur prison et provoquant par leurs cris de désespoir, leurs clameurs féroces, les promeneurs, les passants, les étrangers indignés de ce spectacle et nos jeunes 
écoliers sortant de leur classe qui venaient par groupes se démoraliser à ce déplorable passe-temps. ${ }^{8}$

Les aliénés resteront à Corsier de 1832 à 1838, date à laquelle fut inauguré l'asile des Vernets sur les plans de l'hospice Wakefield en Angleterre. Cet asile qui a valu les observations critiques des Annales médico-psychologiques de 1881 a été finalement remplacé en novembre 1900 par les établissements de Bel-Air.

Comme la psychiatrie française, la psychiatrie genevoise s'est achoppée au problème que lui présentait la justice, notamment celui des actes déraisonnables dont les auteurs paraissent posséder la raison. Elle a eu une affaire qui pourrait être comparée à l'affaire Rivière, dans le sens où il fut évident que la nosographie de l'époque, basée sur les troubles cognitifs de la raison, de l'entendement, bloquait toute explication et compréhension d'un acte où apparaissaient des troubles affectifs de la volonté ${ }^{\text {. }}$

L'affaire J. a éclatée en 1868. Il s'agit d'une garde-malade accusée d'avoir empoisonné à l'atropine huit malades confiés à ses soins. Le juge d'instruction de la République et Canton de Genève a demandé à trois médecins de se prononcer sur l'état mental de la détenue. Voici quelques extraits du rapport:

«Marie J. est malade depuis plusieurs années; les symptômes multiples énumérés par elle se rapportent à un tempérament hystérique dont les principales manifestations ont été, d'après son récit: paralysie des extrémités et des sphincters, crise nerveuse avec perte partielle ou complète de connaissance pendant un temps plus ou moins long (quelques minutes ou quelques heures).»

Suit la description d'autres symptômes de type fonctionnel pour arriver à la conclusion:

«Un examen de près de deux heures ne nous permet de découvrir chez la détenue aucune anomalie de son état mental; en particulier, elle n'a paru atteinte d'aucune hallucination pendant son séjour à la prison. ${ }^{10}$ Signé: Drs Olivet, Badan et Duval*.

Marie J. fut condamnée aux travaux forcés. Elle fut reconnue responsable; pas d'hallucinations, pas de délire, pas de folie. Les médecins psychiatres n'étaient pas prêts à considérer comme pathologiques des comportements qui n'étaient pas délirants. Il y eut cependant un psychiatre qui s'est opposé à cette expertise. Le Dr Auguste Châtelain de Préfargier a fait un reproche de légèreté aux juges et médecins de Genève. Son avis était que «Marie J. est aliénée et, par conséquent, non responsable de ses actes.» Il n'était pas encore question de responsabilité restreinte.

* Le Dr Olivet fut le premier professeur de clinique psychiatrique de la Faculté de Médecine de Genève (d'après la loi du 5 juin 1886), et le Dr Badan était médecin des prisons. 
Il est intéressant de noter quelques changements dans le Code Pénal du Canton de Genève de 1874. L'article 52 concernant l'aliénation mentale mentionne «qu'il n'y a pas d'infraction lorsque l'accusé ou le prévenu était en état d'aliénation mentale au moment où le fait incriminé a eu lieu ou s'il a été contraint par une force à laquelle il n'a pu résister». Le législateur a substitué aliénation mentale à démence. Par ailleurs, on peut se demander si la deuxième possibilité mentionnée dans l'article de loi, c'est-à-dire la force à laquelle l'individu ne peut résister, n'a pas changé de sens depuis 1810. Un examen de la jurisprudence genevoise pourrait déterminer si la force en question était dans l'esprit du législateur une manière d'inclure ces «impulsions insolites» dont a parlé Hoffbauer, la «monomanie»d'Esquirol, «l'impulsion irrésistible» des psychiatres américains.

Les psychiatres au $\mathrm{XIX}^{\mathrm{e}}$ et au début du $\mathrm{XX}^{\mathrm{e}}$ siècle, étaient considérés avec une certaine suspicion par les magistrats. En fait, si le psychiatre était demandé pour une expertise, son pouvoir était grand, car s'il déclarait un prévenu irresponsable, le rôle de la justice était terminé; la personne irresponsable échappait des mains de la justice. Les psychiatres de leur côté étaient très sensibles à l'image qu'ils présentaient. Ainsi, le Dr A.Forel regrettait en 1902 que le Procureur lors d'un procès ait voulu «ridiculiser les aliénistes »11 et le Dr Lamunière constatait que «l'expert aliéniste n'est appelé qu'avec une certaine méfiance $» .{ }^{12}$

Certains experts psychiatres adoptaient alors une attitude diplomatique. Ainsi, le Dr Lamunière préconisait des cours communs aux candidats médecins et aux avocats stagiaires et pensait que "pour faire plus ample connaissance avec les difficultés et problèmes de l'expertise psychiatrique, il serait nécessaire que le juriste puisse se rendre compte de l'évolution de la psychiatrie et connaître jusqu'où vont le pouvoir et le savoir de l'expert, de telle manière qu'il y adapte son questionnaire, c'est-à-dire les questions du juge d'instruction ». ${ }^{13}$

Le Dr Forel ira encore plus loin dans sa diplomatie. Ainsi a-t-il voulu montrer que s'il était préoccupé par la reconnaissance de la compétence du psychiatre auprès du tribunal, ce n'était pas dans le but de soustraire à la justice des délits et des crimes et élargir le domaine de la psychiatrie, mais plutôt dans le but de protéger la société. Cela n'allait pas sans quelque ambiguité. D’une part, il se donnait une peine énorme pour expliquer à ses concitoyens qu'il n'y a pas d'un côté les fous et de l'autre les non-fous, qu'il n'y a pas de limite claire entre la responsabilité et l'irresponsabilité, qu'on peut être responsable concernant un aspect de son comportement et pas un autre, bref qu'il y a divers degrés de responsabilité. D’un autre côté, il essayait de rassurer la justice en écrivant: «qui dit responsabilité limitée, dit les trois quarts du temps, danger social 
augmenté». Et plus loin: «ce n'est pas pour libérer les gens plus rapidement qu'il faut les déclarer partiellement responsables». ${ }^{14}$ Il notait dans le même sens, «qu'on finisse donc une fois pour toutes avec ces querelles byzantines de savoir si tel idiot moral est trop idiot pour mériter la prison de force ou trop peu idiot pour pouvoir être mis à l'asile des aliénés. On se renvoie la balle et on néglige le principal». ${ }^{15}$ Le principal pour lui était qu'on «enferme ces bêtes féroces dans un asile agricole ou industriel, car ils sont forts et pas complètement fous». Forel recommandait que ce genre d'asiles soient sous surveillances juridique et psychiatrique et qu'on enlève à ce genre d'asile tout caractère infamant, rappelant une condamnation judiciaire. Dans son optique, si la surveillance médicale humanise, cela ne nuit pas forcément à la sauvegarde de l'ordre public. Forel montrait ainsi son sens civique, sa préoccupation de l'ordre social. Il avait conscience de son mandat et il l'assumait.

Si le contenu du savoir psychiatrique a évolué, si on a nommé psychopathes ou sociopathes les criminels que l'on appelait idiots moraux dans le temps, la position du psychiatre concernant les délinquants mentalement perturbés n'a pas tellement changée et la sécurité le préoccupe toujours. A la seule différence que cette préoccupation, d'ouverte qu'elle était dans le passé, est occultée actuellement. En fait, la question à laquelle s'exposait et s'expose toujours le psychiatre, lorsqu'il déclare une responsabilité restreinte pour une personne considérée comme dangereuse pour la sécurité publique, est la suivante: où va-t-on mettre ces gens?

Le concept de mesure, caractéristique de l'école de défense sociale, n'avait pas encore fait son apparition dans le code pénal suisse. Cependant, les idées de Forel conduisaient à cela. Il est intéressant de montrer comment il rationalisait l'internement de ceux qu'il décrivait comme «une catégorie d'individus pervers de par leur cerveau anormal qui infestent notre société», individus anormaux qui «ne supportent pas la liberté complète d'action». Voici son raisonnement:

«La liberté des citoyens les plus honnêtes et les plus laborieux est nécessairement limitée à chaque pas par les besoins sociaux. L'ouvrier est enfermé à la fabrique - avec la liberté trop souvent de mourir de faim s'il en sort. Le mineur est enfermé au fond de sa mine; l'infirmier avec les fous et les malades dans l'asile ou à l'hôpital. Le servage du soldat à la légion étrangère est pire souvent que la prison. Que de femmes mariées et d'enfants dans certaines institutions sont plus enfermés et endurent légalement plus de souffrances que les criminels à la maison de force. $\aleph^{16}$

Forel en déduisait qu'il était hypocrite de vitupérer contre la tyrannie pénitentiaire, et de fermer les yeux devant les autres injustices.

Si Forel avait raison dans l'absolu, il nous semble toutefois que ces arguments qui prétendent que la société est une prison, que la prison se trouve partout, 
ignorent et occultent deux fonctions qui différencient ceux qui sont enfermés en prison ou en maison de correction des femmes au foyer, des soldats en caserne et des mineurs sous terre. C'est que les pénitenciers ou établissements spéciaux se caractérisent, contrairement aux autres situations contraignantes, par le fait que leurs clients sont là, parce qu'ils ont commis des actes que la société condamne, et pour être punis. Les détenus savent bien cela ou, en tous cas, on le leur fait sentir.

En 1900, l'asile des Vernets fut remplacé par les établissements de Bel-Air. Le problème du placement des aliénés criminels ne manqua pas de préoccuper la direction. Des thèses de médecine se rapportent à ce problème qui est lié à l'expertise psychiatrique. Les psychiatres de Bel-Air voyaient arriver des prisons, soit de l'Evêché, soit de Saint-Antoine, des criminels aliénés. Le Dr Borel écrivait:

«Tant que ce sera au jury de trancher la question des responsabilités, tant que ce dernier s'attribuera des compétences en aliénation mentale sans recourir à l'examen fait par l'aliéniste, nous verrons se renouveler des condamnations d'aliénés. 》 $^{17}$

Le Dr Kiriakoff constatait que si la prison préventive (Saint-Antoine) envoie trois fois plus de personnes que la pénitentiaire (l'Evêché), ce qui montrait que le Parquet et l'avocat tenaient compte de l'état mental des prévenus, il n'en restait pas moins que la question de la responsabilité devrait être soulevée plus fréquemment, car on trouvait trop de condamnés aliénés ${ }^{18}$.

On constate aussi que le placement des délinquants mentalement perturbés posait des problèmes quasi-identiques à ceux qui se présentent de nos jours. Comme l'érivait le Dr Borel:

«La tendance moderne de transformer peu à peu l'asile en maison ouverte, met à l'écart toute une catégorie d'aliénés et parmi ceux-ci se placent au premier rang les aliénés criminels qui, le plus souvent, sont relégués dans les quartiers agités à cause des désordres qu'ils provoquent au milieu des malades tranquilles ou des tentatives d'évasion qu'ils ne font que trop souvent. ${ }^{19}$

Les différents médecins étaient unanimes pour conclure qu'il faut des asiles spéciaux pour les aliénés criminels. Déjà en 1900 , le criminel aliéné est rejeté de l'asile comme de la prison; le jeu de balle venait de commencer.

Les concepts psychiatriques et juridiques ont certes beaucoup évolué de nos jours. Toutefois, les problèmes épistémologiques et déontologiques de la relation entre psychiatrie et justice n'ont pas fondamentalement changé ${ }^{20}$. On retrouve chez les psychiatres les mêmes préoccupations qu'il y a un siècle, lorsqu'il s'agit de répondre aux questions sur la responsabilité de l'inculpé, ainsi qu'à la nouvelle question concernant l'application des mesures de sûreté. 
Tableau chronologique de la psychiatrie à Genève

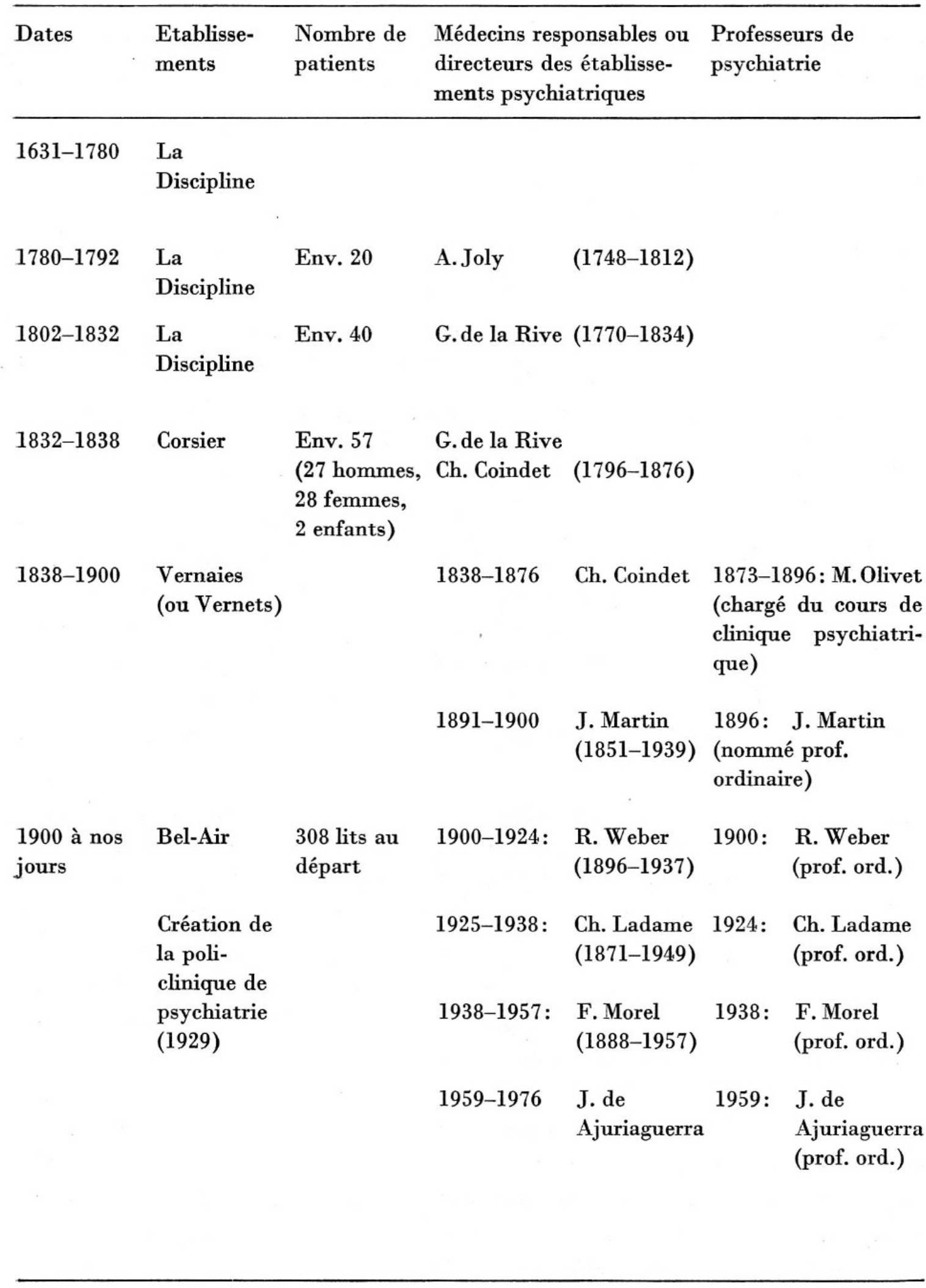


Les fous étaient enfermés avec des condamnés de droit commun; la plupart du temps enchaînés.

A. Joly fait cesser l'emploi des chaînes.

G. de la Rive supprime les fauteuils de force, les tourniquets, les bains de surprise, etc.

Les malades disposent de vastes chambres et se trouvent à la campagne.

Cet hospice qui, au début, était considéré comme exemplaire, fut vite encombré.

Mode de la clinothérapie.

Mode du traitement moral.

1925: débuts de la sismothérapie (insuline, Cardiozol, électrochocs).

1951: débuts des neuroleptiques, des techniques psychothérapeutiques et de l'organisation de l'extrahospitalier.
1770: Le pouvoir administratif est chargé de la surveillance des aliénés.

1810: art. 64 du code pénal français sur l'irresponsabilité des malades mentaux.

1838: Loi sur le placement et la surveillance des aliénés (protège l'aliéné contre la société, mais surtout la société contre l'aliéné).

1895: Création du Conseil de Surveillance des aliénés.

1936: Loi sur le régime des personnes atteintes d'affections mentales On ne parle plus d'aliénés mais de malades. Certificat médical nécessaire à l'internement.

1942: Entrée en vigueur du code pénal suisse et de la notion de responsabilité restreinte. 
Notes

${ }^{1}$ Pour un aperçu historique classique, voir: Pinatel, V., Traité de droit pénal et de criminologie, Tome III, Dalloz, Paris 1975.

2 Archives de l'Etat de Genève, Procès criminels (P.C.), No 11310, 22 novembre 1764, pièce No 80 .

3 Procès criminels, No 18675,23 janvier 1798.

${ }^{4}$ Cramer, M., Un précurseur de Pinel: Abraham Joly, in: Actes de la Société helvétique des sciences naturelles 1974, p.165-167.

${ }^{5}$ Zurbuchen, G. W., Prisons de Genève, Etat de Genève, 1977, p. 68.

6 Jur. pénale X, No 157, 18 novembre 1851.

7 Pour un aperçu de l'histoire de la psychiatrie à Genève, voir: de Morsier, G., Histoire de la psychiatrie et de la neurologie à Genève, in: Gesnerus 34 (1977), fasc. 1/2, p.186-202.

8 Ibid, p. 188.

${ }^{9}$ Cf. Foucault, M. (ed.), Moi, Pierre Rivière..., Gallimard/Juillard, Paris 1973.

10 Jur. pénale X, 572-578, P.C. 1868, P. 52.

${ }^{11}$ Forel, A., Crime et anomalies mentales constitutionnelles. La plaie sociale des déséquilibrés à responsabilité diminuée, Kündig, Genève 1902, p. 221.

12 Lamunière, M., L'affaire Macaland, in: Revue pénale suisse 1909, p. 361-371.

13 Ibid, p. 371.

14 Forel, A., op. cit., p. 287.

15 Ibid, p. 33.

16 Ibid, p. 129.

17 Borel, E., Du placement des aliénés criminels en Suisse, Thèse No 50, Université de Genève, 1904, p. 28.

18 Kiriakoff-Rasoumoff, N., Psychose et emprisonnement, Thèse No 377, Université de Genève, 1912, p. 3.

19 Borel, E., op. cit., p.7.

${ }^{20}$ Parmi les ouvrages les plus intéressants qui traitent de ces problèmes voir: Castel, R., L'ordre psychiatrique, Les Editions de Minuit, Paris 1976; Landry, M., Le psychiatre au tribunal, Privat, Toulouse 1976; Szasz, T., La loi, la liberté et la psychiatrie, Payot, Paris 1977.

\section{Summary}

Legal and psychiatric concepts are constantly evolving. However, the epistemological and ethical problems involved in the working relationships between psychiatry and justice have not fundamentally changed. Nowadays one encounters among psychiatrists the same preoccupations that were current more than a century ago when it comes to assessing the responsibility of a delinquent or his dangerousness. This article presents some examples of the nascent relations between psychiatry and justice in Geneva.

Mme Cléopâtre Montandon, Ph. D.

chargée de recherche

Institut de médecine légale de l'Université

8, passage de la Radio

1211 Genève 4 